\title{
Clinical features and outcomes of JAK2V617F-positive polycythemia vera and essential thrombocythemia according to the JAK2 V617F allele burden
}

\author{
A-Jin Lee ${ }^{1}$, Sang-Gyung Kim ${ }^{1}$, Jun Yeb Nam², Jaehum Yun ${ }^{2}$, Hun-Mo Ryoo ${ }^{2}$, Sung Hwa Bae ${ }^{2}$ \\ ${ }^{1}$ Department of Laboratory Medicine, ${ }^{2}$ Division of Hematology/Oncology, Department of Internal Medicine, Daegu Catholic \\ University Hospital, Daegu Catholic University School of Medicine, Daegu, Korea
}

p-ISSN 2287-979X / e-ISSN 2288-0011 https://doi.org/10.5045/br.2021.2021089 Blood Res 2021;56:259-265.

Received on April 26, 2021 Revised on August 3, 2021 Accepted on September 8, 2021

\author{
Correspondence to \\ Sung Hwa Bae, M.D., Ph.D. \\ Division of Hematology/Oncology, \\ Department of Internal Medicine, Daegu \\ Catholic University Hospital, Daegu \\ Catholic University School of Medicine, \\ 33, Duryugongwon-ro 17-gil, Nam-gu, \\ Daegu 42472, Korea \\ E-mail: sunghwa@cu.ac.kr \\ (C) 2021 Korean Society of Hematology
}

\begin{abstract}
Background
$J A K 2$ mutation status is a well-known risk factor for thrombosis in patients with myeloproliferative neoplasms. However, the clinical usefulness of $J A K 2 \mathrm{~V} 617 \mathrm{~F}$ allele burden is under investigation.
\end{abstract}

\section{Methods}

We retrospectively evaluated the impact of the $J A K 2 \mathrm{~V} 617 \mathrm{~F}$ allele burden on clinical characteristics and outcomes of $J A K 2 \mathrm{~V} 617 \mathrm{~F}$-positive polycythemia vera (PV) and essential thrombocythemia (ET). The $J A K 2 \mathrm{~V} 617 \mathrm{~F}$ allele burden was measured using sequencing.

Results

Altogether, 127 patients with $J A K 2 \mathrm{~V} 617 \mathrm{~F}$ mutation (PV, $\mathrm{N}=61 ; \mathrm{ET}, \mathrm{N}=66$ ) were included in this study. JAK2 V617F allele burdens were positively correlated with white blood cell counts, hemoglobin values, lactate dehydrogenase levels, and platelet counts. The median values of $J A K 2 \mathrm{~V} 617 \mathrm{~F}$ allele burden in patients with $\mathrm{PV}$ and $\mathrm{ET}$ were $58 \%$ and $30 \%$, respectively. A $J A K 2 \mathrm{~V} 617 \mathrm{~F}$ allele burden of $\geq 30 \%$, older age, and a higher hemoglobin level were risk factors for thrombotic events in ET. In patients with PV, older age was the only thrombotic risk factor. The 8-year probabilities of overall survival (OS) were $82.9 \%$ in all patients. A high $J A K 2 \mathrm{~V} 617 \mathrm{~F}$ allele burden $(\geq 58 \%)$ was associated with poor OS in patients with PV. For the patients with ET, the difference in 8-year OS based on the $J A K 2$ V617F allele burden was not significant.

\section{Conclusion}

The $J A K 2 \mathrm{~V} 617 \mathrm{~F}$ allele burden was correlated with hematologic parameters and clinical outcomes. Assessing the $J A K 2 \mathrm{~V} 617 \mathrm{~F}$ allele burden can be helpful in predicting the thrombotic risk and disease course in patients with JAK2 V617F-positive PV and ET.

Key Words JAK2, Thrombosis, Polycythemia, Thrombocythemia, Essential thrombocythemia, Polycythemia vera

\section{INTRODUCTION}

Myeloproliferative neoplasms (MPNs) are characterized by clonal proliferation of hematopoietic progenitors, and include polycythemia vera (PV), essential thrombocythemia (ET), and myelofibrosis (MF). At the time of initial diagnosis, more than half of the patients with PV or ET have no symptoms and show an indolent course [1-3]. The primary characteristics of both diseases are high risks of thrombosis, hemorrhage, myelofibrotic transformation, and leukemic trans- formation [1-5]. Thrombosis is known to be fatal and affects the event-free survival and overall survival in patients with MPNs. Therefore, one of the treatment goals for these diseases is to mitigate the risk of thrombosis $[1-4,6,7]$. The Janus kinase 2 (JAK2) V617F mutation, caused by point mutation, was identified in almost all $B C R-A B L 1$-negative MPNs in 2005 [8-11]. This point mutation was the substitution of phenylalanine for valine in $J A K 2$ codon 617 , and it was named $J A K 2$ V617F. The $J A K 2$ V617F mutation was detected most frequently in PV patients, with approximately $90 \%$ of patients with PV and nearly $50 \%$ of all patients with 
ET and MF carrying the mutation [8-11]. The $J A K 2$ V617F mutation is considered the main driver mutation in $B C R$ - $A B L$-negative MPNs. MPN driver mutations affect the risk of thrombosis, with the $J A K 2 \mathrm{~V} 617 \mathrm{~F}$ mutation being a strong risk factor for thrombosis in patients with ET [4, 12]. Several studies have demonstrated that among MPNs, the $J A K 2 \mathrm{~V} 617 \mathrm{~F}$ allele burden correlates with laboratory hematologic characteristics and clinical endpoints [3, 13-19]. However, there is no definite conclusion regarding the effect of the $J A K 2 \mathrm{~V} 617 \mathrm{~F}$ allele burden on clinical outcomes in patients with PV or ET.

In this study, we measured the JAK2 V617F allele burden and correlated the laboratory parameters and clinical characteristics in patients with $J A K 2 \mathrm{~V} 617 \mathrm{~F}$-positive chronic phase MPNs in a Korean population. We investigated whether the $J A K 2 \mathrm{~V} 617 \mathrm{~F}$ allele burden is related to the clinical phenotype at diagnosis, and aimed to identify the role of $J A K 2$ V617F allele burden as a predictor of complications that occur during disease progression.

\section{MATERIALS AND METHODS}

\section{Patients}

A total of 189 patients newly diagnosed with PV ( $\mathrm{N}=64)$ or ET $(\mathrm{N}=125)$ were retrospectively identified between January 2008 and December 2018 at Daegu Catholic University Hospital. Among them, 127 patients had the JAK2 V617F mutation, comprising 61 patients (95.3\%) with PV and 66 patients $(52.8 \%)$ with ET. Patients with JAK2 V617F-negative MPN or JAK2 V617F-positive PMF were excluded. We evaluated whether they met the criteria for the 2016 WHO classification for the included patients [20]. The main clinical and laboratory data at the time of initial diagnosis of PV or ET were collected, including age, sex, symptoms, cardiovascular risk factors, hemoglobin $(\mathrm{Hb})$ level, hematocrit (Hct) level, white blood cell (WBC) count, platelet count, lactate dehydrogenase (LDH) level, JAK2 V617F allele burden, and bone marrow examination. Disease-related complications, such as thrombosis and bleeding events, myelofibrotic transformation or leukemic transformation, secondary neoplasm, and live/death were reviewed. The study was conducted in accordance with the Declaration of Helsinki and was approved by the Institutional Review Board of Daegu Catholic University Hospital (IRB No. CR-19-111). The need for informed patient consent was waived due to the retrospective nature of the analysis.

\section{Molecular analysis}

DNA extraction from peripheral blood samples was performed using a QIAamp DNA Mini Kit (Qiagen, Hilden, Germany) according to the manufacturer's instructions. DNA was amplified using PCR primers (forward primer: 5' AGC AAGCTTTCTCACAAGCA 3', reverse primer: 5' CTGACA CCTAGCTGTGATCCTG 3'). Amplified PCR products were sequenced using the Big Dye Terminator Cycle Sequencing kit (Applied Biosystems, Waltham, MA, USA) and an ABI 3130 Genetic Analyzer (Applied Biosystems). The values of the $J A K 2 \mathrm{~V} 617 \mathrm{~F}$ allele burden, which was the ratio of mutant allele to total allele, were obtained by direct sequencing.

\begin{tabular}{|c|c|c|c|}
\hline & Total $(\mathrm{N}=127)$ & $\begin{array}{l}J A K 2 \mathrm{~V} 617 \mathrm{~F} \text { mutation } \mathrm{PV} \\
\qquad(\mathrm{N}=61)\end{array}$ & $\begin{array}{c}J A K 2 \mathrm{~V} 617 \mathrm{~F} \text { mutation } \mathrm{ET} \\
\qquad(\mathrm{N}=66)\end{array}$ \\
\hline Age at diagnosis, yr & $69(27-88)$ & $64(37-88)$ & $69(27-86)$ \\
\hline Sex, female & $75(59.1 \%)$ & $31(50.8 \%)$ & $44(66.7 \%)$ \\
\hline \multicolumn{4}{|l|}{ Cardiovascular risk factors } \\
\hline Diabetes mellitus & $22(17.3 \%)$ & $12(19.7 \%)$ & $10(15.2 \%)$ \\
\hline Hypertension & $67(52.8 \%)$ & $32(52.5 \%)$ & $35(53.0 \%)$ \\
\hline Dyslipidemia & $10(7.9 \%)$ & $6(9.8 \%)$ & $4(6.1 \%)$ \\
\hline Smoking, current or ex-smoker & $5(4.0 \%)$ & $4(6.5 \%)$ & $1(1.5 \%)$ \\
\hline WBC $\left(\times 10^{9} / \mathrm{L}\right)$ & $13.4(4.2-45.4)$ & $17.4(5.1-45.4)$ & $12.3(4.2-45.0)$ \\
\hline $\mathrm{Hb}(\mathrm{g} / \mathrm{dL})$ & $15(8-22)$ & $18(11-22)$ & $13\left(8^{-17)}\right.$ \\
\hline Hct $(\%)$ & $46(24-68)$ & $56(36-68)$ & $39(24-54)$ \\
\hline Platelet $\left(\times 10^{9} / \mathrm{L}\right)$ & $700(95-1,934)$ & $540(95-1,490)$ & $930(465-1,934)$ \\
\hline $\mathrm{LDH}(\mathrm{U} / \mathrm{L})$ & $462(205-1,141)$ & $453(215-1,141)$ & $464(205-929)$ \\
\hline JAK2 V617F allele (\%) & $40.0(5.0-100.0)$ & $58.0(5.0-100.0)$ & $30.0(5.0-100.0)$ \\
\hline Thrombosis & $51(40.2 \%)$ & $17(27.9 \%)$ & $34(51.5 \%)$ \\
\hline At dusgnosis & $44(34.6 \%)$ & $14(23.0 \%)$ & $30(45.5 \%)$ \\
\hline During follow up ${ }^{b)}$ & $20(15.6 \%)$ & $7(11.5 \%)$ & $13(19.7 \%)$ \\
\hline Progression to MF & $5(3.9 \%)$ & $3(4.9 \%)$ & $2(3.0 \%)$ \\
\hline
\end{tabular}

${ }^{a}$ Data are presented as median (range) or $\mathrm{N}(\%) .{ }^{\text {b) }}$ Median follow-up duration was 71.3 months.

Abbreviations: ET, essential thrombocythemia; LDH, lactate dehydrogenase; MF, myelofibrosis; MPN, myeloproliferative neoplasm; PV, polycythemia vera; WBC, white blood cell. 


\section{Statistical analysis}

Data were analyzed using the Mann-Whitney U test for quantitative values and the Chi-square test for categorical data. Correlations between JAK2 V617F allele burden and clinical and laboratory parameters were determined using Pearson's product-moment correlation. Multivariate logistic regression analysis was performed to test the impact of different covariates on the risk of thrombosis. All tests were two-sided, and the type I error level was set at 0.05 . Univariate logistic regression analysis was used to identify the risk factors. Parameters with $P<0.2$ were identified by stepwise backward elimination, and further multivariate logistic regression analysis was performed to investigate the independent risk factors. Overall survival (OS) and thrombosis-free survival (TFS) were estimated using the KaplanMeier method, and differences in survival were compared using the log-rank test. OS was defined as the period from MPN diagnosis to death from any cause, while TFS was defined as the period from MPN diagnosis to death or the development of new thrombosis. TFS was analyzed for all patients regardless of whether they had thrombosis at the time of initial diagnosis. Statistical analysis was performed using SPSS software version 18 (SPSS Inc., Chicago, IL, USA) and $\mathrm{R}$ version 4.0.0.

\section{RESULTS}

\section{Patient characteristics}

A total of 127 patients with PV $(\mathrm{N}=61)$ or ET $(\mathrm{N}=66)$, of whom 52 (40.9\%) were male and 75 (59.1\%) were female, were included in this study. The median age at initial diagnosis was 69 years (range, 27-88 yr). The median JAK2 V617F allele burden in the included patients was $40.0 \%$, ranging from $5.0 \%$ to $100.0 \%$. The median $J A K 2$ V617F allele burden was $58.0 \%$ and $30.0 \%$ in patients with PV and ET, respectively (Table 1). Median follow-up duration was 71.3 months (range, 1.0-288.1 mo). Sixty-four thrombotic events were observed in 51 patients $(40.2 \%)$ at the time of diagnosis and during the follow-up period, including 17 patients with PV and 34 patients with ET. Cerebrovascular thrombosis $(\mathrm{N}=50)$ was the most prevalent event, followed by coronary

Table 2. Clinical and laboratory characteristics in patients with polycythemia vera according to JAK2 V617F allele burden.

\begin{tabular}{lccc} 
& JAK2 V617F allele burden $<58 \%(\mathrm{~N}=30)$ & $J A K 2 \mathrm{~V} 617 \mathrm{~F}$ allele burden $\geq 58 \%(\mathrm{~N}=31)$ & $P$ \\
\hline Age at diagnosis, yr & $65(37-88)$ & $63(45-80)$ & 0.880 \\
Sex, females & $13(43.3 \%)$ & $18(58.1 \%)$ & 0.371 \\
$\mathrm{WBC}\left(\times 10^{9} / \mathrm{L}\right)$ & $13.0(5.1-27.5)$ & $19.0(9.5-45.4)$ & 0.002 \\
$\mathrm{Hb}(\mathrm{g} / \mathrm{dL})$ & $18(11-22)$ & $18(13-22)$ & 0.569 \\
$\mathrm{Hct}(\%)$ & $56(36-68)$ & $56(42-65)$ & 0.297 \\
Platelet $\left(\times 10^{9} / \mathrm{L}\right)$ & $551(221-1,490)$ & $513(95-1,321)$ & 0.902 \\
$\mathrm{LDH}(\mathrm{U} / \mathrm{L})$ & $420(215-940)$ & $498(248-1,141)$ & 0.065 \\
Thrombosis & & & 0.937 \\
At diagnosis & $9(30.0 \%)$ & $5(16.1 \%)$ & \\
$\quad$ During follow up & $1(3.3 \%)$ & $5(16.1 \%)$ & 1.000 \\
Progression to MF & $1(3.3 \%)$ & $2(6.5 \%)$ & \\
\hline
\end{tabular}

Data are presented as median (range) or $\mathrm{N}(\%)$. $P$-value was determined by Mann-Whitney $\mathrm{U}$ test or Chi-square test.

Abbreviations: $\mathrm{LDH}$, lactate dehydrogenase; MF, myelofibrosis; PV, polycythemia vera; WBC, white blood cell.

Table 3. Clinical and laboratory characteristics in patients with essential thrombocythemia according to JAK2 V617F allele burden.

\begin{tabular}{lcrc} 
& $J A K 2$ V617F allele burden $<30 \%(\mathrm{~N}=34)$ & $J A K 2$ V617F allele burden $\geq 30 \%(\mathrm{~N}=32)$ & $P$ \\
\hline Age at initial diagnosis, $y r$ & $68(27-82)$ & $72.5(49-86)$ & 0.003 \\
Sex, females & $22(64.7 \%)$ & $22(68.8 \%)$ & 0.931 \\
$\mathrm{WBC}\left(\times 10^{9} / \mathrm{L}\right)$ & $11.1(4.2-30.3)$ & $14.0(6.7-45.0)$ & 0.035 \\
$\mathrm{Hb}(\mathrm{g} / \mathrm{dL})$ & $13(8-17)$ & $13(9-17)$ & 0.949 \\
$\mathrm{Hct}(\%)$ & $39(24-49)$ & $39(31-54)$ & 0.380 \\
$\mathrm{Platelet}\left(\times 10^{9} / \mathrm{L}\right)$ & $859(465-1,934)$ & $966(517-1,729)$ & 0.420 \\
$\mathrm{LDH}(\mathrm{U} / \mathrm{L})$ & $391(205-872)$ & $514.5(240-929)$ & 0.049 \\
Thrombosis & $11(32.4 \%)$ & $23(71.9 \%)$ & 0.003 \\
At diagnosis & $10(29.4 \%)$ & $20(62.5 \%)$ & $9(28.1 \%)$ \\
$\quad$ During follow up & $4(11.8 \%)$ & $2(6.2 \%)$ & 0.446 \\
Progression to MF & $0(0.0 \%)$ & & \\
\hline
\end{tabular}

Data are presented as median (range) or $\mathrm{N}(\%) . P$-value was determined by Mann-Whitney $\mathrm{U}$ test or Chi-square test.

Abbreviations: ET, essential thrombocythemia; LDH, lactate dehydrogenase; MF, myelofibrosis; WBC, white blood cell. 
artery thrombosis $(\mathrm{N}=6)$, other peripheral artery thrombosis $(\mathrm{N}=5)$, and venous thromboembolism or pulmonary embolism $(\mathrm{N}=5)$. Forty-four (34.6\%) patients experienced thrombosis at the time of diagnosis, while 20 patients (15.6\%) experienced thrombotic events after diagnosis. Of the 44 patients who had thrombotic events at the time of diagnosis, 13 had recurrent thrombosis during the follow-up period. Myelofibrotic transformation occurred in 3 patients with PV and 2 patients with ET. The risk of myelofibrotic transformation was not significantly related to the $J A K 2$ V617F allele burden.

We stratified the patients into two groups according to the median value of $J A K 2 \mathrm{~V} 617 \mathrm{~F}$ allele burden in both the $\mathrm{PV}$ and ET groups and compared the results between each group. In $\mathrm{PV}$, patients with $\geq 58 \%$ allele burden had higher WBC counts $(P=0.002)$ (Table 2). In ET, the higher $J A K 2$ V617F allele burden group ( $\geq 30 \%)$ was older $(P=0.003)$, had higher WBC counts $(P=0.035)$, and had a higher incidence $(71.9 \%)$ of thrombosis $(P=0.003)$ (Table 3$)$.

\section{Risk factors associated thrombosis}

Thrombotic events detected at MPN diagnosis or during the follow-up period were observed in $27.9 \%$ (17/61) and $51.5 \%(34 / 66)$ of patients with PV and ET, respectively. The factors associated with thrombosis, determined via univariate logistic regression analysis, are shown in Table 4. Multivariate logistic regression analysis showed that older age [odds ratio (OR), 1.07; 95\% confidence interval (CI), 0.98-1.18] was associated with a higher incidence of thrombosis in patients with PV (Table 5). In patients with ET, older age (OR, 1.36; 95\% CI, 1.14-1.72), higher hemoglobin (OR, 1.26; 95\% CI, 1.09-1.54), and V617F allele burden $\geq$ $30 \%$ (OR, 1.03; 95\% CI, 1.00-1.08) were identified as risk factors for thrombosis (Table 5).

\section{Survival}

The median follow-up duration for live patients was 71.3 months (range, 1.0-288.1 mo). Within this time frame, 12 patients died of any cause: 3 due to pneumonia, 2 due to cerebral infarction, 2 due to ischemic heart disease, 2 due to gastrointestinal bleeding, 1 due to cancer, 1 due to trauma, and 1 due to unknown cause. The 8-year cumulative incidence of thrombosis was $43.9 \%$ and $21.3 \%$ in ET and PV patients, respectively. The 8-year probabilities of OS and TFS were $82.9 \%$ and $67.3 \%$, respectively. OS and TFS did not significantly differ between PV and ET patients with JAK2 V617F mutation (8-yr OS, $78.0 \%$ vs. $92.7 \%$, respectively, $P=0.475$; 8 -yr TFS, $69.9 \%$ vs. $64.7 \%$, respectively, $P=0.419$ ).

Among the PV patients, the 8-year OS difference was determined between patients with $\geq 58 \% J A K 2$ V617F allele burden and those with $<58 \%$ allele burden $(67.3 \%$ vs. $100.0 \%$, respectively, $P=0.048$ ). Of the 61 patients with $J A K 2$ mutant $\mathrm{PV}$, seven patients died, with six out of the seven having $\geq 58 \% J A K 2$ V617F mutant alleles at the time of enrollment in the study. The 8-year TFS of PV patients with $J A K 2 \mathrm{~V} 617 \mathrm{~F}$ allele burden $\geq 58 \%$ was $53.5 \%$, and that of patients with $<58 \% J A K 2$ allele burden was $100 \%$ $(P=0.006)$.

For the patients with ET, the 8-year OS in patients with $\geq 30 \%$ and $<30 \% J A K 2$ V617F allele burden were $92.2 \%$ and $96.2 \%$, respectively $(P=0.349)$. TFS in patients with $\geq$ $30 \%$ and $<30 \% J A K 2$ V617F allele burden were $62.0 \%$ and $65.3 \%$, respectively $(P=0.416)$ (Fig. 1$)$.

Table 5. Results of multivariate logistic regression analysis for thrombosis.

\begin{tabular}{lccc} 
& Odds ratio & $95 \% \mathrm{Cl}$ & $P$ \\
\hline PV & & & \\
$\quad$ Age at diagnosis & 1.10 & $1.00-1.21$ & 0.045 \\
ET & & & \\
$\quad$ Age at diagnosis & 1.08 & $1.01-1.15$ & 0.022 \\
Hb & 1.79 & $1.10-2.91$ & 0.019 \\
JAK2 V617F allele burden & 1.05 & $1.00-1.10$ & 0.039
\end{tabular}

Abbreviations: $\mathrm{Cl}$, confidence interval; $\mathrm{ET}$, essential thrombocythemia; $\mathrm{PV}$, polycythemia vera.

Table 4. Results of univariate logistic regression analysis for thrombosis.

\begin{tabular}{|c|c|c|c|c|c|c|}
\hline & \multicolumn{3}{|c|}{$P V(N=61)$} & \multicolumn{3}{|c|}{ ET $(N=66)$} \\
\hline & Odds ratio & $95 \% \mathrm{Cl}$ & $P$ & Odds ratio & $95 \% \mathrm{Cl}$ & $P$ \\
\hline Age at diagnosis, yr & 1.08 & $1.01-1.15$ & 0.018 & 1.03 & $0.99-1.07$ & 0.106 \\
\hline Sex, females & 0.58 & $0.19-1.81$ & 0.351 & 1.58 & $0.56-4.46$ & 0.385 \\
\hline$J A K 2 \mathrm{~V} 617 \mathrm{~F}$ allele $(\%)$ & 1.00 & $0.98-1.03$ & 0.742 & 1.05 & $1.01-1.08$ & 0.006 \\
\hline WBC $\left(\times 10^{9} / \mathrm{L}\right)$ & 1.00 & $1.00-1.00$ & 0.514 & 1.00 & $1.00-1.00$ & 0.671 \\
\hline $\mathrm{Hb}(\mathrm{g} / \mathrm{dL})$ & 0.87 & 0.69-1.09 & 0.222 & 1.26 & $0.98-1.63$ & 0.069 \\
\hline Hct $(\%)$ & 0.95 & $0.88-1.03$ & 0.196 & 1.10 & $1.01-1.21$ & 0.031 \\
\hline Platelet $\left(\times 10^{9} / \mathrm{L}\right)$ & 1.00 & $1.00-1.00$ & 0.135 & 1.00 & $1.00-1.00$ & 0.404 \\
\hline $\mathrm{LDH}(\mathrm{U} / \mathrm{L})$ & 1.00 & $1.00-1.00$ & 0.162 & 1.00 & $1.00-1.01$ & 0.075 \\
\hline
\end{tabular}

Variables with $P<0.2$ were adapted to the multivariate analysis.

Abbreviations: ANC, absolute neutrophil count; ET, essential thrombocythemia; PV, polycythemia vera; WBC, white blood cell. 

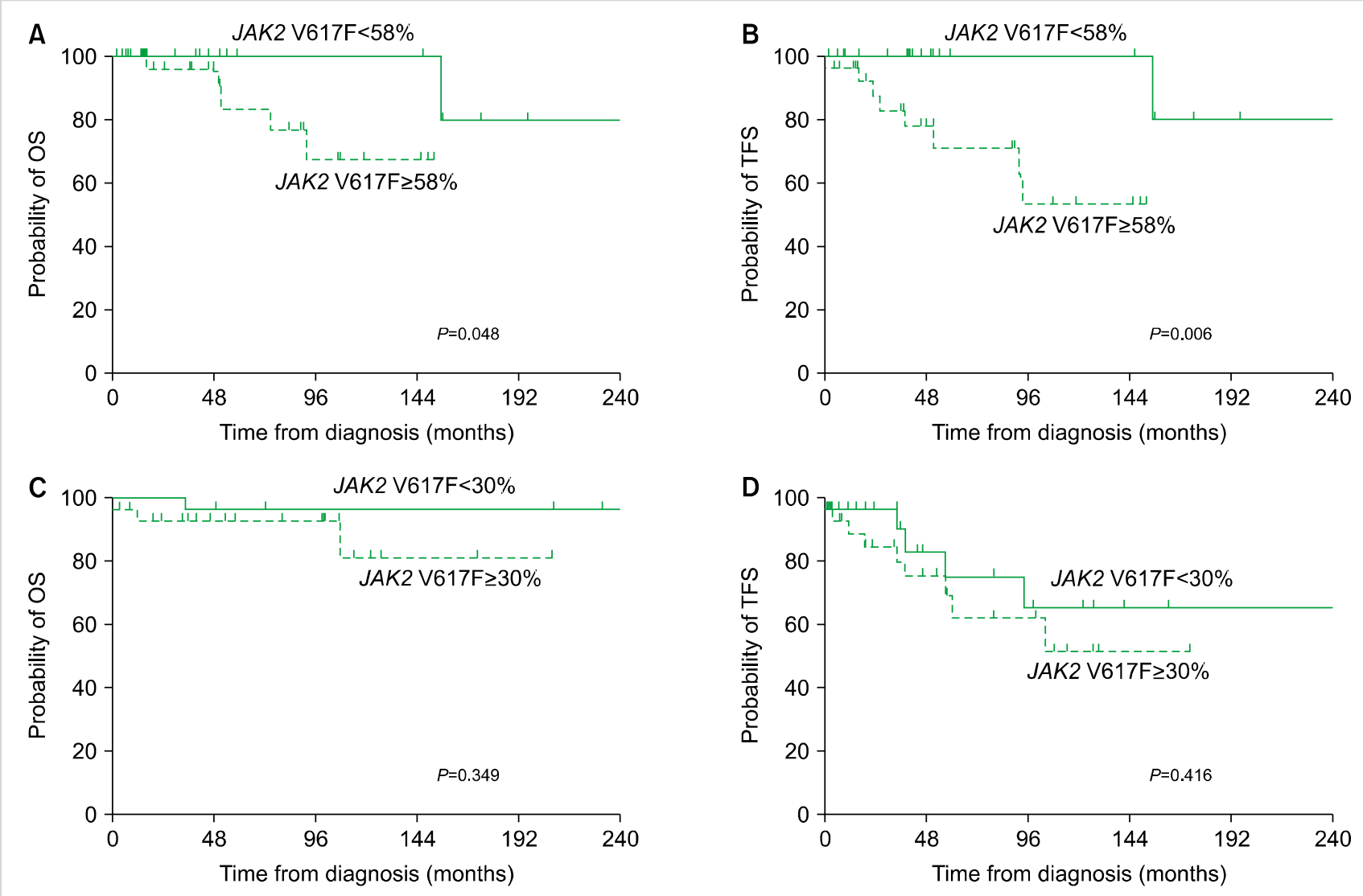

Fig. 1. Survival according to JAK2 V617F allele burden. Overall survival (OS) in patients with PV (A), thrombosis free survival (TFS) in patients with PV (B), OS in patients with ET (C), TFS in patients with ET (D).

\section{DISCUSSION}

The presence of $J A K 2$ V617F mutation is a well-characterized risk factor for thrombotic complications in patients with ET and is used for risk stratification and management $[3,12]$. The clinical usefulness of the $J A K 2$ allele burden, however, is being investigated, and its clinical potential is being evaluated. The results of clinical studies trying to define the relationship between $J A K 2$ V617F mutation burden and the occurrence of vascular complications in ET are conflicting $[3,13,15-18,21]$. In this study, the $J A K 2$ V617F allele burden can be used as a risk factor for thrombosis in patients with $J A K 2$ V617F-positive ET. The results of the present study correspond well with those of the largest previous study, which included 165 patients with mutated ET. Antonioli et al. [21] reported that patients with $\geq 30 \% J A K 2 \mathrm{~V} 617 \mathrm{~F}$ allele burden were associated with a 3.0-times higher relative risk of arterial thrombosis at diagnosis. The impact of the $J A K 2$ V617F allele burden and the JAK2 V617F mutation status on the development of thrombotic events should be evaluated in a large-scale prospective study. Older age and a previous thrombotic event are also well-known risk factors for thrombosis in patients with ET [3]. In this study, the other factors associated with thrombotic events were age and hemoglobin in ET.

In this study, the risk of developing thrombosis in addition to that of myelofibrotic transformation was not significantly related to the $J A K 2 \mathrm{~V} 617 \mathrm{~F}$ allele burden in PV. However, OS was significantly shorter in PV patients with a higher $J A K 2$ mutation burden than in those with a lower JAK2 mutation burden. Several studies have been conducted to determine whether the burden of the JAK2 mutant allele could predict major clinical outcomes in PV. Vannucchi et al. [19] reported that having $\geq 75 \% J A K 2 \mathrm{~V} 617 \mathrm{~F}$ allele burden at the time of PV diagnosis was significantly associated with an increased rate of cardiovascular events and risk of needing chemotherapy during the follow-up period. In contrast, another prospective study showed that they did not identify a relationship between $J A K 2$ mutant allele burden and thrombotic risk in PV. However, they showed that myelofibrosis-free survival was significantly different between PV patients with $<50 \% J A K 2$ V617F allele burden and those with $\geq 50 \%$ mutant allele burden [14]. Many factors, including patient characteristics such as age and comorbidities, disease characteristics such as laboratory findings and genetic alterations, and treatment factors, may influence the long-term outcomes of PV. Therefore, it is difficult to conclude whether $J A K 2$ mutational burden is associated with thrombotic risk, myelofibrotic or leukemic trans- 
formation, or survival. A large prospective trial with standardization of the JAK2 V617F allele burden measurement is needed to clarify this important issue.

Patients with PV had a higher JAK2 V617F allele burden than those with ET. The results of the present study correspond well with those of an earlier study which reported that patients with ET have a lower allele burden among MPN patients, and those with PV have a higher allele burden compared to those with ET [16, 22].

In the present study, the incidence of thrombosis was higher than the reported incidence in previous studies. In this study, thrombotic events included events at diagnosis and events during the follow-up period. Considering the frequency at diagnosis, thrombotic events were found in $43.9 \%$ of ET patients and in $21.3 \%$ of PV patients. This frequency is also higher than the reported frequency in a previous study [4]. The difference might be due to a selection bias stemming from the small number of patients.

This study found that the prevalence of thrombosis was higher in patients with ET than in those with PV. We focused on and analyzed patients with $J A K 2$ V617F mutated ET patients and did not include patients with $J A K 2$ wild type. According to the International Prognostic Score of Thrombosis in World Health Organization-Essential Thrombocythemia (IPSET-thrombosis), which scores JAK2 V617F as 2 points, JAK2 V617F mutated patients can be stratified as intermediate-risk or high-risk [12]. They reported that the intermediate-risk and high-risk groups showed a thrombosis risk of $2.35 \% /$ year and 3.56\%/year, respectively [12].

In this study, the $J A K 2 \mathrm{~V} 617 \mathrm{~F}$ test was performed as part of the routine clinical care. The $J A K 2 \mathrm{~V} 617 \mathrm{~F}$ allele burden was calculated using direct sequencing, and the data were retrospectively reviewed. The sensitivity of direct sequencing is $10-20 \%$, which is considered relatively low [23]. There are several available methods for detecting $J A K 2$ V617F allele burden quantitatively, such as real-time quantitative PCR, pyrosequencing, next-generation sequencing, and droplet digital PCR $[24,25]$. These tests show high sensitivity and can identify an allele burden as low as 1-3\% [24]. Although direct sequencing is not recommended due to its low sensitivity, we retrospectively reviewed the routinely measured data.

This study has limitations due to its small sample size and retrospective nature. In further studies, the $J A K 2$ V617F allele burden needs to be measured prospectively with a more sensitive method for research purposes.

The $J A K 2$ V617F allele burden in patients with PV was higher than that in patients with ET. The $J A K 2$ V617F allele burden was correlated with the hematologic parameters. A high $J A K 2 \mathrm{~V} 617 \mathrm{~F}$ allele burden is a risk factor for thrombosis in ET. A high $J A K 2$ V617F allele burden is associated with a shorter OS in patients with PV. Assessing the $J A K 2$ V617F allele burden can be helpful in predicting the disease course and thrombotic risk in patients with $J A K 2 \mathrm{~V} 617 \mathrm{~F}$-positive PV and ET.

\section{Authors' Disclosures of Potential Conflicts of Interest}

No potential conflicts of interest relevant to this article were reported.

\section{REFERENCES}

1. Hultcrantz M, Kristinsson SY, Andersson TM, et al. Patterns of survival among patients with myeloproliferative neoplasms diagnosed in Sweden from 1973 to 2008: a population-based study. J Clin Oncol 2012;30:2995-3001.

2. Tefferi A, Guglielmelli P, Larson DR, et al. Long-term survival and blast transformation in molecularly annotated essential thrombocythemia, polycythemia vera, and myelofibrosis. Blood 2014;124: 2507-13.

3. Tefferi A, Barbui T. Polycythemia vera and essential thrombocythemia: 2021 update on diagnosis, risk-stratification and management. Am J Hematol 2020;95:1599-613.

4. Rumi E, Pietra D, Ferretti V, et al. JAK2 or CALR mutation status defines subtypes of essential thrombocythemia with substantially different clinical course and outcomes. Blood 2014;123:1544-51.

5. Lee MW, Ryu H, Song IC, Lee HJ, Yun HJ, Jo DY. Outcomes of patients with essential thrombocythemia and unnoticed thrombocytosis prior to diagnosis. Blood Res 2020;55:281-2.

6. Barbui T, Finazzi G, Falanga A. Myeloproliferative neoplasms and thrombosis. Blood 2013;122:2176-84.

7. Rungjirajittranon $T$, Owattanapanich $W$, Ungprasert $P$, Siritanaratkul $\mathrm{N}$, Ruchutrakool T. A systematic review and meta-analysis of the prevalence of thrombosis and bleeding at diagnosis of Philadelphianegative myeloproliferative neoplasms. BMC Cancer 2019;19:184.

8. Baxter EJ, Scott LM, Campbell PJ, et al. Acquired mutation of the tyrosine kinase JAK2 in human myeloproliferative disorders. Lancet 2005;365:1054-61.

9. James C, Ugo V, Le Couédic JP, et al. A unique clonal JAK2 mutation leading to constitutive signalling causes polycythaemia vera. Nature 2005;434:1144-8.

10. Kralovics R, Passamonti F, Buser AS, et al. A gain-of-function mutation of JAK2 in myeloproliferative disorders. N Engl J Med 2005;352:1779-90.

11. Levine RL, Wadleigh M, Cools J, et al. Activating mutation in the tyrosine kinase JAK2 in polycythemia vera, essential thrombocythemia, and myeloid metaplasia with myelofibrosis. Cancer Cell 2005;7:387-97.

12. Barbui T, Finazzi G, Carobbio A, et al. Development and validation of an International Prognostic Score of thrombosis in World Health Organization-essential thrombocythemia (IPSET-thrombosis). Blood 2012;120:5128-33.

13. Vannucchi AM, Pieri L, Guglielmelli P. JAK2 allele burden in the myeloproliferative neoplasms: effects on phenotype, prognosis and change with treatment. Ther Adv Hematol 2011;2:21-32.

14. Passamonti F, Rumi E, Pietra D, et al. A prospective study of 338 patients with polycythemia vera: the impact of JAK2 (V617F) allele burden and leukocytosis on fibrotic or leukemic disease transformation and vascular complications. Leukemia 2010;24: 1574-9. 
15. Borowczyk M, Wojtaszewska M, Lewandowski K, et al. The JAK2 V617F mutational status and allele burden may be related with the risk of venous thromboembolic events in patients with Philadelphia-negative myeloproliferative neoplasms. Thromb Res 2015;135:272-80.

16. Passamonti F, Rumi E. Clinical relevance of JAK2 (V617F) mutant allele burden. Haematologica 2009;94:7-10.

17. Vannucchi AM, Antonioli E, Guglielmelli P, Pardanani A, Tefferi A. Clinical correlates of JAK2V617F presence or allele burden in myeloproliferative neoplasms: a critical reappraisal. Leukemia 2008;22:1299-307.

18. Carobbio A, Finazzi G, Antonioli E, et al. JAK2V617F allele burden and thrombosis: a direct comparison in essential thrombocythemia and polycythemia vera. Exp Hematol 2009;37:1016-21.

19. Vannucchi AM, Antonioli E, Guglielmelli P, et al. Prospective identification of high-risk polycythemia vera patients based on JAK2(V617F) allele burden. Leukemia 2007;21:1952-9.

20. Arber DA, Orazi A, Hasserjian R, et al. The 2016 revision to the
World Health Organization classification of myeloid neoplasms and acute leukemia. Blood 2016;127:2391-405.

21. Antonioli E, Guglielmelli P, Poli G, et al. Influence of JAK2V617F allele burden on phenotype in essential thrombocythemia. Haematologica 2008;93:41-8.

22. Tefferi A, Vainchenker W. Myeloproliferative neoplasms: molecular pathophysiology, essential clinical understanding, and treatment strategies. J Clin Oncol 2011;29:573-82.

23. Bakker E. Is the DNA sequence the gold standard in genetic testing? Quality of molecular genetic tests assessed. Clin Chem 2006;52:557-8

24. Bench AJ, White HE, Foroni L, et al. Molecular diagnosis of the myeloproliferative neoplasms: UK guidelines for the detection of JAK2 V617F and other relevant mutations. Br J Haematol 2013;160:25-34.

25. Kinz E, Leiherer A, Lang AH, Drexel H, Muendlein A. Accurate quantitation of JAK2 V617F allele burden by array-based digital PCR. Int J Lab Hematol 2015;37:217-24. 